TECHNICAL NOTE

\author{
S.P. Ferns \\ J.J. Schneiders \\ M. Siebes \\ R. van den Berg \\ E.T. van Bavel \\ C.B. Majoie
}

\section{Intracranial Blood-Flow Velocity and Pressure Measurements Using an Intra-Arterial Dual- Sensor Guidewire}

\begin{abstract}
SUMmARY: Hemodynamics is thought to play a role in the growth and rupture of intracranial aneurysms. In 4 patients, we obtained local pressure and BFV by using a dual-sensor pressure and Doppler velocity wire within and in vessels surrounding unruptured aneurysms. Local BFVs can serve as boundary conditions for computational fluid dynamics, whereas pressure recordings provide direct information on the mechanical load imposed on the aneurysm. Both measurements may thus add to patient-specific rupture-risk assessment.
\end{abstract}

ABBREVIATIONS: BFV = blood flow velocity; $C F D=$ computational fluid dynamics; ICA = internal carotid artery.
H emodynamics is believed to play an important role in aneurysm growth and rupture. ${ }^{1}$ Hemodynamic profiles have been predicted by using computational methods, suggesting high risk of rupture in aneurysms with disturbed flow patterns, small impingement regions, and narrow jets. ${ }^{1,2}$ However, proper estimation of these flow patterns requires recording of BFVs in the vessels surrounding the aneurysms. ${ }^{3}$ In addition to flow patterns, the pressure pulse inside the aneurysm may also contribute to growth and rupture. ${ }^{4,5}$ Yet, direct recordings of local BFV are limited to MR imagingbased techniques, which, so far, have found little application in intracranial aneurysms, and local measurements of pressure profiles are fully lacking.

Intra-arterial BFV and pressure measurements are used on an increasingly regular basis in coronary interventions by using a dual-sensor pressure and Doppler velocity guidewire (Fig 1). Currently, the primary applications of such measurements serve to assess the hemodynamic consequences of coronary artery stenoses. ${ }^{6}$ We aimed to use this guidewire in intracranial vessels to evaluate this new use for the wire. We simultaneously acquired BFV and pressure signals in multiple locations in patients with unruptured intracranial aneurysms before endovascular treatment.

\section{Description of Techniques}

\section{Patients and Aneurysms}

Four patients $(52,56,64$, and 70 years of age) scheduled to undergo endovascular coiling of an unruptured intracranial aneurysm were invited to participate in this study. Aneurysms were located at the ophthalmic artery $(6 \times 8 \mathrm{~mm})$, the posterior communicating artery $(9 \times 9$ and $6 \times 7 \mathrm{~mm})$, and the supraclinoid internal carotid artery

Received March 20, 2009; accepted after revision April 26.

From the Departments of Radiology (S.P.F., J.J.S., R.v.d.B., C.B.M.) and Biomedical Engineering and Physics (E.T.v.B., M.S.), Academic Medical Center, University of Amsterdam, the Netherlands.

ComboWires used in this study were sponsored by Volcano Therapeutics, Europe Headquarters, Zaventem, Belgium.

Please address correspondence to S.P. Ferns, MD, Department of Radiology, G1, Academic Medical Center, University of Amsterdam, PO Box 22660, 1100 DD Amsterdam, the Netherlands; e-mail: s.p.ferns@amc.uva.nl

DOl 10.3174/ajnr.A1718
( $3 \times 5 \mathrm{~mm}$ ). The local ethics committee approved the study, and written informed consent was obtained from all participants.

\section{Instrumentation}

A 0.014-inch dual-sensor pressure and Doppler velocity wire (ComboWire; Volcano Corporation, Rancho Cordova, California) was used in combination with matching hardware (ComboMap pressure and flow system). The caliber and material of the ComboWire are identical to the standard guidewire used for endovascular treatment. Shaping of the tip for better navigation is possible but may harm its delicate sensors. To avoid potential added risk of adverse events (thrombus formation, perforation) caused by its straight tip, we did not use the ComboWire for navigation purposes. The wire tip was carefully positioned to avoid interference from the vessel wall. The velocity waveform comprises the temporal sequence of the maximum of fluid velocity within a cone-shaped beam $5 \mathrm{~mm}$ from the tip. Pressure measurements were considered valid when clear systole-diastole profiles were visible. Possible drift of the pressure signal intensity could be resolved by re-zeroing. All pressure and velocity signals were recorded at $200 \mathrm{~Hz}$.

\section{Flow-Velocity and Pressure Measurements}

All procedures were performed with the patient under general anesthesia with full systemic heparinization on a single-plane angiographic unit (Integris Allura Neuro; Philips Medical Systems, Best, the Netherlands) following institutional protocol. The recordings also included the electrocardiogram and pressure derived from an intra-arterial pressure catheter in the radial artery.

A $5 \mathrm{~F}$ guiding catheter was positioned in the vessel harboring the aneurysm, and the standard microcatheter was navigated beyond and within the aneurysm by using a standard microguidewire. Subsequently, the microguidewire was withdrawn and replaced by the ComboWire. All measurements were performed in the ICA, distal to the aneurysm, proximal to the aneurysm, and at the cervical level, consecutively. If aneurysm geometry and size allowed safe catheterization, measurements were also performed within the aneurysm. The exact locations were recorded with roadmap control. At each location, we recorded simultaneous BFV and pressure waveforms for a minimum of 10 consecutive cardiac cycles. All hemodynamic measurements took approximately 15 minutes and were completed before coil placement. 


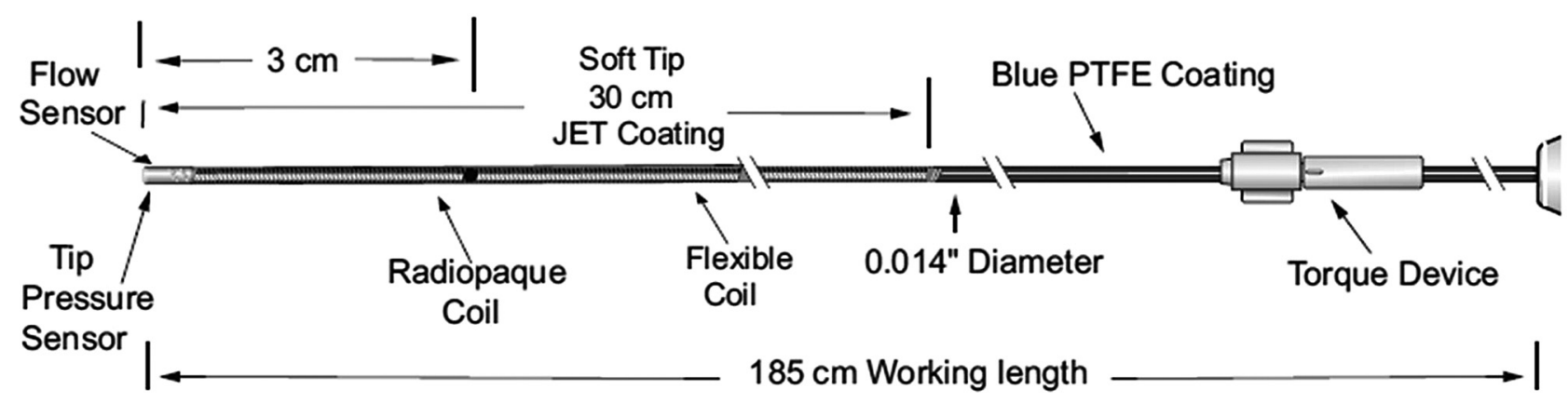

Fig 1. Detail of the ComboWire. Reprinted with permission of Volcano Therapeutics. PTFE indicates polytetrafluoroethylene.

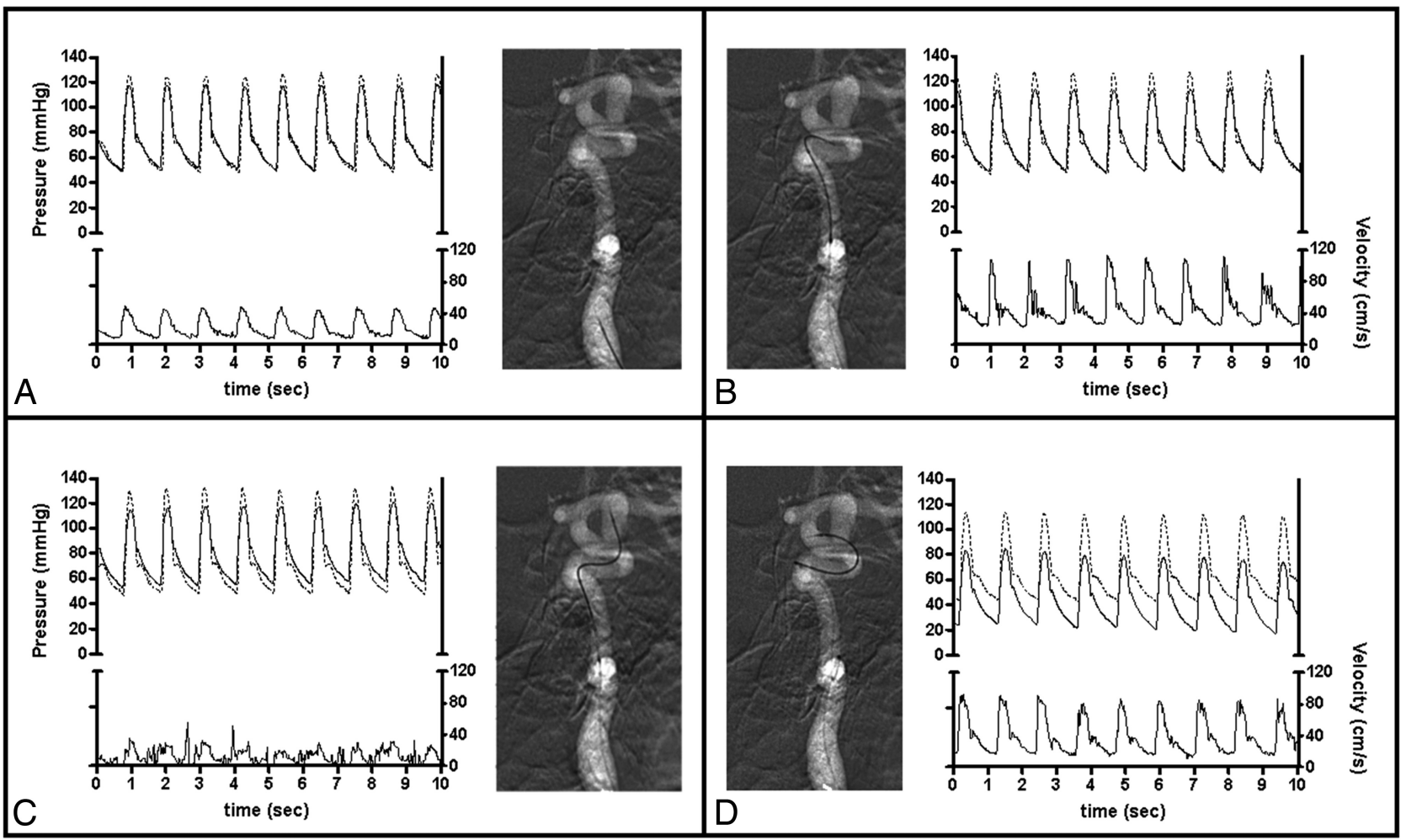

-.... $\mathrm{Pa}(\mathrm{mmHg})-\mathrm{Pd}(\mathrm{mmHg})$

Fig 2. BFV and pressure measurements using the ComboWire in the ICA at the cervical level $(A)$, proximal to the aneurysm $(B)$, intra-aneurysmally $(C)$, and distal to the aneurysm $(D)$. The graphs show matching flow velocity and pressure waveforms extracted from the ComboMap recordings. The upper part of each panel depicts pressure (mm Hg): systemic pressure measured in the radial artery (Pa) and intracranial pressure measured at the tip of the wire (Pd). The lower part of the graphs show flow velocity (centimeters per second). Sec indicates seconds.

\section{Data Analysis}

The guidewire could not be positioned in 2 of 16 locations (12.5\%) due to unfavorable geometry. The recorded raw data were extracted for further analysis. Figure 2 illustrates typical results in 1 patient: a poorly defined BFV pattern within the aneurysm, a lower maximal BFV at the cervical level compared with other locations, and stable pressure pulses (systolic minus diastolic pressure) in all locations. Averaging pressure pulses measured in the different locations resulted in a mean of $65.8 \pm 9.2 \mathrm{~mm} \mathrm{Hg}$ in patient $1 ; 65.4 \pm 2.59 \mathrm{~mm} \mathrm{Hg}$ in patient 2; $48.3 \pm 3.1 \mathrm{~mm} \mathrm{Hg}$ in patient 3; and $48.8 \pm 1.5 \mathrm{~mm} \mathrm{Hg}$ in patient 4 .

Figure 3 shows maximal BFV measured in all patients. An increase of maximal BFV proximal to the aneurysms compared with the cervical level of the ICA was seen in all patients (range, 11.5-71.4 cm/s). Measurements distal to the aneurysm were successful in 3 patients and failed in 1. Compared to proximal, BFV distal to the aneurysm remained stable in 2 patients and decreased in 1 patient.

\section{Discussion}

We have successfully obtained simultaneous measurements of BFV and pressure in intracranial aneurysms and surrounding vessels by using a dual-sensor pressure and Doppler velocity wire (ComboWire). BFV was higher proximal to aneurysms compared with BFV at the cervical level of the ICA, consistent with the decreasing caliber of the upstream ICA. These data indicate that wall shear stress was enhanced proximal to the aneurysm. Pressure pulses were comparable between all intracranial locations within patients.

Noninvasive techniques for measuring flow velocities have been developed by using cardiac-gated 3D phase-contrast MR 


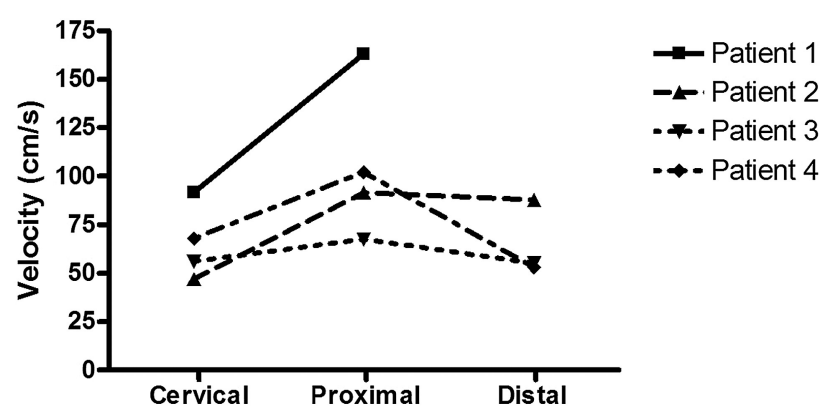

Fig 3. Maximal BFV in centimeters per second measured with the ComboWire in the ICA at the cervical level (Cervical), proximal to the aneurysm (Proximal), and distal to the aneurysm (Distal). Intra-aneurysmal measurements are not plotted here because we considered them to be less accurate (see "Data Analysis"). Maximal BFV was calculated by averaging 5 consecutive peaks of the ComboMap flow signal. One measurement is lacking because the wire could not be placed distal to the aneurysm in patient 1 due to unfavorable geometry.

imaging. ${ }^{7}$ Unfortunately, prolonged MR imaging is usually not suitable in acutely ill patients with ruptured aneurysms. Benndorf et $\mathrm{al}^{8}$ have previously demonstrated the feasibility of flow-velocity measurements within giant intracranial aneurysms with a Doppler guidewire in 2 patients. We were not able to obtain clearly defined BFV measurements within aneurysms. The size and shape of the conebeam used for velocity recording makes this technique unsuitable for direct use inside the small- to medium-sized aneurysms of our study. When measuring inside an aneurysm, large spatial gradients in velocity magnitude and direction occur in the sampling volume. These lead to an envelope of maximal velocities during the heart cycle, which is difficult to interpret. We only used the ComboWire in the ICA with relatively large caliber, to diminish vessel wall interference and to obtain reliable BFV and pressure profiles. It remains to be determined whether measurements are also feasible in smaller caliber middle cerebral, anterior cerebral, and vertebrobasilar arteries.

We encountered additional difficulties using the ComboWire. Obtaining a good Doppler flow signal intensity could take several minutes because of the relatively small caliber of tortuous vessels. Wire-vessel wall interference hampered pressure measurements. The added risk to patients is negligible when the ComboWire is not used for navigation and is inserted only via the microcatheter previously deployed with a standard microguidewire. Possible risks of using the ComboWire with a straight tip for navigation may be damage of the vessel wall with possible subsequent thrombus formation and perforation of the vessel or aneurysm wall.

The capability of the ComboWire to obtain simultaneously intra-arterial pressure and BFV provides a valuable novel way for determining patient-specific hemodynamics. The simulation of fluid flows by using CFD allows the prediction of hemodynamics, but lack of accurate flow-velocity measurements has hampered the study of hemodynamics of intracranial aneurysms. ${ }^{1,2}$ For future construction of patientspecific CFD models, we are now able to provide BFV proximal and distal to the aneurysm. The pressure recordings provide additional information on the mechanical load imposed on the aneurysm. Both measurements may be vital in the individual rupture-risk assessment of patients with an unruptured aneurysm.

\section{Acknowledgment}

We thank H. Gratama van Andel for his extensive help with the measurements.

\section{References}

1. Cebral JR, Castro MA, Burgess JE, et al. Characterization of cerebral aneurysms for assessing risk of rupture by using patient-specific computational hemodynamics models. AJNR Am J Neuroradiol 2005;26:2550-59

2. Castro MA, Putman CM, Cebral JR. Patient-specific computational fluid dynamics modeling of anterior communicating artery aneurysms: a study of the sensitivity of intra-aneurysmal flow patterns to flow conditions in the carotid arteries. AJNR Am J Neuroradiol 2006;27:2061-68

3. Venugopal P, Valentino D, Schmitt H, et al. Sensitivity of patient-specific numerical simulation of cerebral aneurysm hemodynamics to inflow boundary conditions. J Neurosurg 2007;106:1051-60

4. Hashimoto T. Flow velocity studies in vein pouch model aneurysms. Neurol Res 1993;15:185-91

5. Chatzipromodrou I, Tricoli A, Poulikanos D, et al. Haemodynamics and wall remodelling of a growing cerebral aneurysm: a computational model. J Biomech 2007;40:412-26. Epub 2006 Mar 9

6. Siebes M, Verhoeff BJ, Meeuwissen M, et al. Single-wire pressure and flow velocity measurement to quantify coronary stenosis hemodynamics and effects of percutaneous interventions. Circulation 2004;109:756-62

7. Meckel S, Stalder AF, Santini F, et al. In vivo visualization and analysis of 3-D hemodynamics in cerebral aneurysms with flow-sensitized 4-D MR imaging at 3 T. Neuroradiology 2008;50:473-84. Epub 2008 Mar 19

8. Benndorf G, Wellnhofer E, Lanksch W, et al. Intraaneurysmal flow: evaluation with Doppler guidewires. AJNR Am J Neuroradiol 1996;17:1333-37 